\title{
Basic FATE (Focus Assessed Transthoracic Echocardiography)
}

FATE is the original focused echo protocol practiced since 1989. FATE is easily and quickly learned and can be applied in all clinical scenarios e.g.: pre-, per-, and postoperative, intensive care medicine, emergencies, resuscitation, ambulances, inflight - no limitations, ect. FATE can be performed even with the patient in the sitting position. FATE also encounters a quick guidance to interpret the echocardiographic findings and to put it into the clinical context.

\section{Two FATE levels exist}

$\nabla$

- Basic FATE. Based on two-dimensional imaging of the easiest cardiac views including M-mode.

- Advanced FATE. Basic FATE supplied with extended cardiac views and Doppler ultrasound for cardiac output and pressure measurement together with assessment of LV diastolic function.

\section{Objective}

$\nabla$

Provide the attendees with basic echocardiographic needs for the non-cardiologist in order to guide cardiopulmonary optimization. All theory is based on E-learning and the modules must be completed before the course.

\section{Who can attend?}

$\nabla$

Physicians from all specialities e.g.: anaesthesiologists, intensivists, emergency physicians, internal medicine physicians, surgeons, general practitioners.

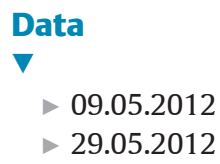

$$
\begin{aligned}
& \text { 13.08.2012 } \\
& \text { - } 05.11 .2012 \\
& \text { - 26.11.2012 }
\end{aligned}
$$

Venue. Aarhus University Hospital, Brendstrupgaardsvej 100, Skejby, 8200 Aarhus N, Denmark

\begin{tabular}{|c|c|}
\hline From / To & \\
\hline 10:00-10:15 & Introduction, coffee \\
\hline 10:15-10:30 & $\begin{array}{l}\text { Highlights from E-learning, } \\
\text { coffee }\end{array}$ \\
\hline 10:30-11:45 & HOT 1 (FATE views) \\
\hline $11: 45-12: 00$ & $\begin{array}{l}\text { Highlights from HOT } 1 \text { and E- } \\
\text { learning }\end{array}$ \\
\hline 12:00-13:00 & $\begin{array}{l}\text { HOT } 2 \text { (FATE views, M-mode } \\
\text { in pos } 3 \text {, parasternal long axis } \\
\text { view) }\end{array}$ \\
\hline 13:00-13:30 & Lunch (included) \\
\hline 13:30-13:45 & $\begin{array}{l}\text { Highlights from HOT } 2 \text { and E- } \\
\text { learning }\end{array}$ \\
\hline $13: 45-14: 45$ & $\begin{array}{l}\text { HOT } 3 \text { (Repetition of HOT } \\
1-2, \text { M-mode for MAPSE and } \\
\text { TAPSE, extended views) }\end{array}$ \\
\hline 14:45-15:00 & $\begin{array}{l}\text { Highlights from HOT } 3 \text { and } \\
\text { introduction to certification } \\
\text { and scenario training }\end{array}$ \\
\hline 15:00-15:15 & Break with coffee \\
\hline 15:15-17:00 & $\begin{array}{l}\text { HOT } 4 \text { (Certification and } \\
\text { scenario training, discussion, } \\
\text { new trends and evaluation) }\end{array}$ \\
\hline
\end{tabular}

\section{Programme \\ $\nabla$}

\section{Pre-course qualification}

No prior experience is required. Language. English, if any non-Danish-speaking participants.

Pre course E-learning modules are under development and will be implemented when convenient.

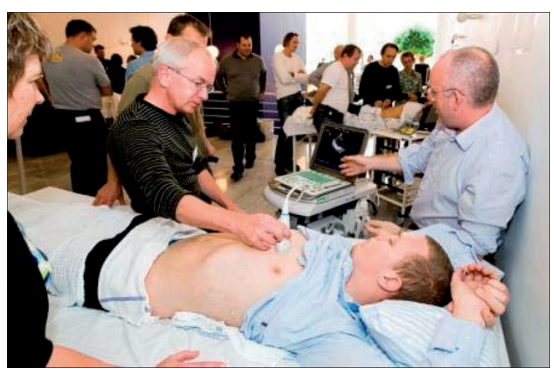

\section{Course convenor} $\nabla$

Professor Erik Sloth, Department of Anaesthesia and Intensive Care, Aarhus University Hospital, Skejby, having more than 20 years experience with focus assessed transthoracic echocardiography (TOE and TTE).

Fee. 400 Euro including access to E-learning modules and lunch.

A certificate will be issued upon completion of the course.

\section{Registration}

$\nabla$

E-Mail. bianjens@rm.dk

Tel.. (+ 45) 22580136

See. http.//www.fate-protocol.com

http.//www.usabcd.org 